\title{
Simulation for Texture Formation of Both Face-Centered-Cubic Metals and Body-Centered-Cubic Ones Based on Rotational Symmetry among Principal Axes
}

\author{
Hiroaki Masui \\ Department of Engineering, Teikyo University, Utsunomiya-shi, Japan
}

Email address:

masuihiro@jcom.home.ne.jp

To cite this article:

Hiroaki Masui. Simulation for Texture Formation of Both Face-Centered-Cubic Metals and Body-Centered-Cubic Ones Based on Rotational Symmetry among Principal Axes. Advances in Materials. Special Issue: Academic Research for Multidisciplinary.

Vol. 7, No. 2, 2018, pp. 34-43. doi: 10.11648/j.am.20180702.14

Received: May 10, 2018; Accepted: May 30, 2018; Published: July 11, 2018

\begin{abstract}
Based on the rotational symmetry of the principal axes of X [100], Y [010] and Z [001], in fcc metal 24 possible combinations of the five slips on $\{111\}$ planes on $<110>$ direction while in bcc metal 72 possible combinations of the five slips on $\{110\}$ planes on $<111>$ direction by intersection of two kinds of $\{110\}$ planes from the three ones composed of $\{110\},\{101\}$ and $\{011\}$ are respectively chosen both based on Taylor's formidable restriction rule of the five slips. In fcc metal, orientation at onset (minimum) of Taylor factor $M$ value, i.e. the minimum total slip amount, shows the cube $\{100\}<001>$ and the $M$ value gradually increases by way of $\{100\}<001>\rightarrow\{100\}<016>\rightarrow\{100\}<013>\rightarrow\{100\}<012>\rightarrow\{100\}<023>\rightarrow\{100\}<0,9,11>$ with decrease of $\varphi_{1}$ or does $\{100\}<001>\rightarrow\{016\}<100>\rightarrow\{013\}<100>\rightarrow\{0,6,13\}<100>$ with increase of $\varphi_{2}$, most of which were experimentally reported as indiscrete recrystallized orientations with lowest dislocation density named the cluster composed of cube and cube-family in fcc metal. In bec metal, crystal rotation is carried out by only one solution among the 72 by the minimum total slip amount at every strain and simulates properly lengthy of accumulated researcher's experimental results such as the three stable orientations of bec metal in rolling $\{112\}<110>,\left\{\begin{array}{lll}11 & 11\end{array}\right\}<4411>$ and $\{100\}<011>$.
\end{abstract}

Keywords: Body-Centered-Cubic, Face-Centered-Cubic, Deformation, Texture, Symmetry

\section{Introduction}

The principal axes of $\mathrm{X}$ [100], Y [010] and Z [001] are perpendicular to each other as the three orbits of $\{ \pm X\},\{ \pm Y\}$ and $\{ \pm Z\}$ by a rotational symmetry of mathematical group theory in such way that component $\mathrm{X}$ is not related to $\mathrm{Y}$ or $\mathrm{Z}$ one another whichever [1].There is a conservation quantity in the symmetry [2]. For example, a small ball at the top of Mexican hat may drop down on the bottom everywhere equally and symmetrically within $360^{\circ}$ around the top due to existence of potential energy of the ball as a conservation quantity. Once the hat inclined, the ball would lose both the quantity and symmetry. As Taylor proved, crystal rotates so that slips occur associating themselves with the minimum total slip amount [3]. The minimum total slip amount in crystal by Taylor corresponds to both the conservation quantity in the rotational symmetry of cubic crystal and even Taylor factor $M$ value itself of the material.
These approaches will be useful for both face centered cubic (fcc) metal and body centered cubic (bcc) metal.

\section{Model}

\subsection{Fcc Metal}

Here, $\mathrm{dX}_{\mathrm{i}}$ and $\mathrm{dY} \mathrm{Y}_{\mathrm{i}}$ mean pair glides with common glide direction on neighbor planes in fcc metal and $a, b, c, d$ are synthetic glide of them respectively. Figure 1 shows eight shears in four pairs of $<110>$ direction on $\{111\}$ plane around principal axis $Z$ [001] in fcc metal. Similarly there are another eight shears in four pairs on both $\{111\}$ planes around $X$ [100] and Y [010] axes, which consequently satisfy the twelve glide systems of fcc metal equally. Relation among the eight shears $\mathrm{dX}_{1}, \mathrm{dY}_{1}, \mathrm{dX}_{2}, \mathrm{dY}_{2}, \mathrm{dX}_{3}, \mathrm{dY}_{3}, \mathrm{dX}_{4}, \mathrm{dY}_{4}$ and both of strain 
tensor components $\mathrm{d} \varepsilon_{11}, \mathrm{~d} \varepsilon_{22}, \mathrm{~d} \varepsilon_{33}, \mathrm{~d} \varepsilon_{12}, \mathrm{~d} \varepsilon_{13}, \mathrm{~d} \varepsilon_{23}$ and rotation $\mathrm{d} \varphi_{1}, \mathrm{~d} \varphi_{2}, \mathrm{~d} \varphi_{3}$ around the principal axes X [100], Y [010] and Z [001] noted as 1, 2 and 3 respectively can be deduced as shown in (1) for Z [001] group from geometrical relation in Figure 1.

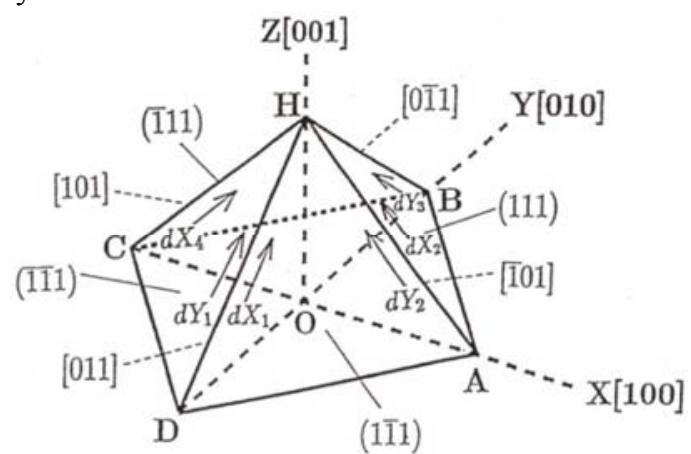

Figure 1. Illustration of the $\{111\}<110>$ glide systems in the $Z$ ([001]) group and the principal axes for fcc metal in the present model.

$$
\begin{aligned}
\mathrm{dX}_{1}= & (\sqrt{ } 6 / 8)\left(\mathrm{d} \varepsilon_{33}-\left(\mathrm{d} \varepsilon_{22}-\mathrm{d} \varepsilon_{11}\right)+2 \mathrm{~d} \varepsilon_{12}+4 \mathrm{~d} \varepsilon_{13}+2 \mathrm{~d} \varepsilon_{23}-2 \mathrm{~d} \varphi_{3}+2 \mathrm{~d} \varphi_{1}\right) \\
\mathrm{dX}_{2} & =(\sqrt{ } 6 / 8)\left(\mathrm{d} \varepsilon_{33}+\left(\mathrm{d} \varepsilon_{22}-\mathrm{d} \varepsilon_{11}\right)-2 \mathrm{~d} \varepsilon_{12}-2 \mathrm{~d} \varepsilon_{13}+4 \mathrm{~d} \varepsilon_{23}-2 \mathrm{~d} \varphi_{3}+2 \mathrm{~d} \varphi_{2}\right) \\
\mathrm{dX}_{3} & =(\sqrt{ } 6 / 8)\left(\mathrm{d} \varepsilon_{33}-\left(\mathrm{d} \varepsilon_{22}-\mathrm{d} \varepsilon_{11}\right)+2 \mathrm{~d} \varepsilon_{12}-4 \mathrm{~d} \varepsilon_{13}-2 \mathrm{~d} \varepsilon_{23}-2 \mathrm{~d} \varphi_{3}-2 \mathrm{~d} \varphi_{1}\right) \\
\mathrm{dX}_{4} & =(\sqrt{ } 6 / 8)\left(\mathrm{d} \varepsilon_{33}+\left(\mathrm{d} \varepsilon_{22}-\mathrm{d} \varepsilon_{11}\right)-2 \mathrm{~d} \varepsilon_{12}+2 \mathrm{~d} \varepsilon_{13}-4 \mathrm{~d} \varepsilon_{23}-2 \mathrm{~d} \varphi_{3}-2 \mathrm{~d} \varphi_{2}\right) \\
\mathrm{dY}_{1} & =(\sqrt{ } 6 / 8)\left(\mathrm{d} \varepsilon_{33}-\left(\mathrm{d} \varepsilon_{22}-\mathrm{d} \varepsilon_{11}\right)-2 \mathrm{~d} \varepsilon_{12}-4 \mathrm{~d} \varepsilon_{13}+2 \mathrm{~d} \varepsilon_{23}+2 \mathrm{~d} \varphi_{3}+2 \mathrm{~d} \varphi_{1}\right) \\
\mathrm{dY}_{2} & =(\sqrt{ } 6 / 8)\left(\mathrm{d} \varepsilon_{33}+\left(\mathrm{d} \varepsilon_{22}-\mathrm{d} \varepsilon_{11}\right)+2 \mathrm{~d} \varepsilon_{12}-2 \mathrm{~d} \varepsilon_{13}-4 \mathrm{~d} \varepsilon_{23}+2 \mathrm{~d} \varphi_{3}+2 \mathrm{~d} \varphi_{2}\right) \\
\mathrm{dY}_{3} & =(\sqrt{ } 6 / 8)\left(\mathrm{d} \varepsilon_{33}-\left(\mathrm{d} \varepsilon_{22}-\mathrm{d} \varepsilon_{11}\right)-2 \mathrm{~d} \varepsilon_{12}+4 \mathrm{~d} \varepsilon_{13}-2 \mathrm{~d} \varepsilon_{23}+2 \mathrm{~d} \varphi_{3}-2 \mathrm{~d} \varphi_{1}\right) \\
\mathrm{dY}_{4} & =(\sqrt{ } 6 / 8)\left(\mathrm{d} \varepsilon_{33}+\left(\mathrm{d} \varepsilon_{22}-\mathrm{d} \varepsilon_{11}\right)+2 \mathrm{~d} \varepsilon_{12}+2 \mathrm{~d} \varepsilon_{13}+4 \mathrm{~d} \varepsilon_{23}+2 \mathrm{~d} \varphi_{3}-2 \mathrm{~d} \varphi_{2}\right)
\end{aligned}
$$

There are another similar relations for X [100] and Y [010] groups.

To be remarked in relation of $(1)$, rotation $d \varphi_{1}, d \varphi_{2}$ and $d \varphi_{3}$ can be directly deduced by putting either of three components from the eight shears $\mathrm{dX}_{1}, \mathrm{dY}_{1}, \mathrm{dX}_{2}, \mathrm{dY}_{2}, \mathrm{dX}_{3}, \mathrm{dY}_{3}, \mathrm{dX}_{4}, \mathrm{dY}_{4}$ to zero which coincides with Taylor's five shears theory [3] as more concretely demonstrated in 2.2 (bcc metal).

The theory calculates sum of total shears by (2) each in these three groups of $\mathrm{Z}$ [001], X [100] and Y [010] and chooses minimum of them based on Taylor's theory [3] as the minimum total slip amount $\Gamma$ and resultantly selects glide group which achieves maximum work satisfying yield surface of material.

$$
\begin{gathered}
\Gamma=\mathrm{a}+\mathrm{b}+\mathrm{c}+\mathrm{d} \\
=\sqrt{d X_{1}^{2}+d Y_{1}^{2}}+\sqrt{d X_{2}^{2}+d Y_{2}^{2}}+\sqrt{d X_{3}^{2}+d Y_{3}^{2}}+\sqrt{d X_{4}^{2}+d Y_{4}^{2}}
\end{gathered}
$$

Taylor factor $\mathrm{M}$ value has been also obtained as total shears, i.e., the minimum total slip amount $\Gamma$ as shown in (2). In (2), it shall be remarked that if angle between plane whereon $\mathrm{dX}$ and one whereon $\mathrm{dY}$ is $\theta$, there exists, on the other hand, also supplementary angle $180^{\circ}-\theta$ owing to equivalent reverse slip on opposite side. Therefore if we ask average synthetic shear for both, their cosine terms cancel each other in average of the cosine theorem and finally there leaves only $\mathrm{dX}^{2}+\mathrm{dY}^{2}$ term in the average irrespective of angle in fcc, bcc or $\mathrm{NaCl}$ whatever.
It may be important to understand that the $M$ value depends solely both on crystal structure (fcc, bcc, $\mathrm{NaCl}$ ) and deformation style, and doesn't depend on a degree of deformation. Thus, distribution (isosurface map) of Taylor factor $\mathrm{M}$ value in 3D ODF coordinates can be drawn in rolling.

\subsection{Bcc Metal}

There are three kinds of $\{110\}$ planes having a common glide axis $\langle 111\rangle$ in bcc crystal such as those illustrated on Figure 2 in case around Z [001] axis.



Figure 2. Three kinds of $\{110\}$ planes composed of $\{110\},\{101\}$ and $\{011\}$ ones around the principal Z [001] axis with a common slip direction $\langle 111\rangle$ in bcc crystal. 
(a)

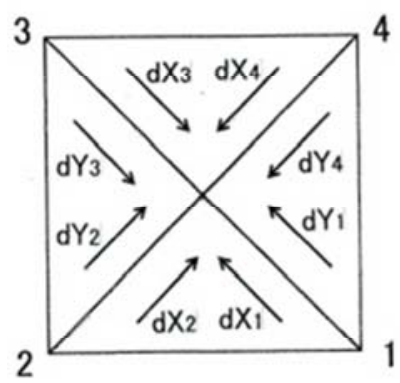

(b)


$x$

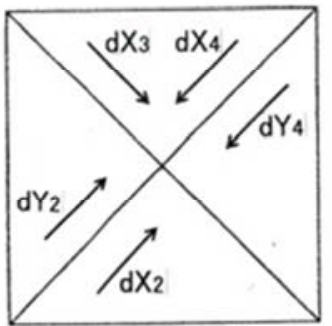

4

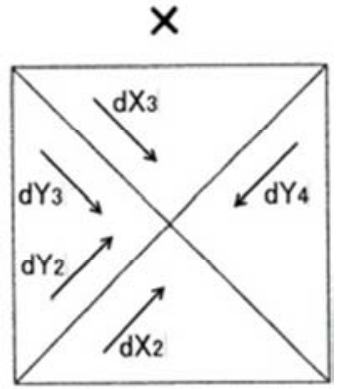

Figure 3. Example of an intersection between $\{101\}$ and $\{011\}$ in Figure 2.

(a) Eight slips in bcc cubic metal wherefrom five slips are needed for deformation and crystal rotation.

(b)Two possible combinations of the five slips ( $(0)$ and two impossible ones $(\times)$ in the model by the rule of the Taylor's formidable restriction of the five slips, "all of which must be chosen so that two shears occur on each of two planes, one on the third and none on the fourth", are exemplified in case of $\mathrm{dX}=\mathrm{dY} \mathrm{Y}_{1}=0$ $\left(\mathrm{dX}_{2}=0\right.$ or $\mathrm{dY}_{4}=0$ is possible).

Calculation method is exemplified as follows.

Firstly, an intersection of two kinds of $\{110\}$ planes from the three ones composed of $\{110\},\{101\}$ and $\{011\}$ as illustrated on Figure 2 is chosen. As example, an intersection between $\{101\}$ and $\{011\}$ in Figure 2 is chosen and shears on $\{101\}$ group planes on four $\langle 111\rangle$ directions in case around $\mathrm{Z}[001]$ axis are named by turns $\mathrm{dX}_{1}, \mathrm{dX}_{2}, \mathrm{dX}_{3}$ and $\mathrm{dX}_{4}$ and those on $\{011\}$ group planes are done $\mathrm{dY}_{1}, \mathrm{dY}_{2}, \mathrm{dY}_{3}$ and $\mathrm{dY}_{4}$ correspondently as shown in Figure 3. These are geometrically correlated to strain components $\mathrm{d} \varepsilon_{11}, \mathrm{~d} \varepsilon_{22}, \mathrm{~d} \varepsilon_{33}$, $\mathrm{d} \varepsilon_{12}, \mathrm{~d} \varepsilon_{13}$ and $\mathrm{d} \varepsilon_{23}$ and crystal rotation $\mathrm{d} \varphi_{1}, \mathrm{~d} \varphi_{2}$ and $\mathrm{d} \varphi_{3}$ around the principal axes $\mathrm{X}, \mathrm{Y}$ and $\mathrm{Z}$ noted as 1,2 and 3 respectively as demonstrated in (3) and accordingly a crystal rotation and texture formation can be calculated for the bcc crystal during any of compression, elongation, rolling and others by putting three of the eight shears to zero.

Second the eight slips, however, must be reduced to five shears according to Taylor's formidable combination (restriction) rule of the five slips as follows [3]. The geometrical condition rule for a given strain cannot be satisfied if the five shears are chosen so that two are taken from one group, i.e. one slip plane, and the remaining three are chosen one from each of the three remaining groups, in other words, all of which must be chosen so that two shears occur on each of two planes, one on the third and none on the fourth. This rule was properly applied to the present model as exemplified in Figure 3. By the Taylor's restriction rule of the five, Figure 3 in case of $\mathrm{dX}_{1}=\mathrm{dY}_{1}=0$ shows two possible combinations $(0)$ of the five slips and two impossible ones $(\times)$.
This phenomenon of two possible combinations on Figure 3 appears in each case of $\mathrm{dXi}=\mathrm{dYi}=0$ for four $\langle 111\rangle$ directions $(\mathrm{i}=1 \sim 4)$, and furthermore every for each group around $\mathrm{X}, \mathrm{Y}$ and $\mathrm{Z}$ axes.

This shows $24(=2 \times 4 \times 3)$ possible combinations in the intersection of two groups of $\{110\}$ planes (in this case $\{101\}$ and $\{011\})$ from the three ones. There are three kinds of intersections of two $\{110\}$ planes as illustrated on Figure 2 and the model accordingly provides with $72(=24 \times 3)$ possible combinations of the five slips as a whole.

In this case of the example between $\{101\}$ and $\{011\}$ from the three kinds of intersections around $Z$ [001] axis in Figure 2, there are relations as demonstrated in (3) among the shears $\mathrm{dX}_{1}, \mathrm{dX}_{2}, \mathrm{dX}_{3}$ and $\mathrm{dX}_{4}$ on $\{101\}$ group planes on four $\langle 111\rangle$ directions as well as $\mathrm{dY}_{1}, \mathrm{dY}_{2}, \mathrm{dY}_{3}$ and $\mathrm{dY}_{4}$ on $\{011\}$ group planes, and strain components, crystal rotations. Similar equations exist also in each case around X [100] and Y [010] cases. Further, applied to (3), a calculation is carried out for crystal rotation in the possible case $(\circ)$ of $\mathrm{dX}_{1}=\mathrm{dY}_{1}=0, \mathrm{dX}_{2}=0$ on Figure 3 according to the Taylor's restriction rule of the five slips and its solution is introduced as in (4). The above example is only one of total 72 possible combinations of the five slips by the three kinds of intersections of two $\{110\}$ planes on $\langle 111\rangle$ directions in bcc metal and each of the 72 has respectively similar equations as (4). Third an actual crystal rotation by (4) proceeds only for the case of which the total slip amount $\Gamma$ (gamma) defined by (2) is minimum among the 72 cases. Calculation of (2) is performed by inserting both equation (3) and (4) to (2). Suppose one of the 
72 cases is selected in the model.

$$
\begin{aligned}
& \mathrm{dX}=(\sqrt{ } 6 / 4)\left(\mathrm{d} \varepsilon_{11}+\mathrm{d} \varepsilon_{12}+\mathrm{d} \varepsilon_{13}-2 \mathrm{~d} \varepsilon_{23}+\mathrm{d} \varphi_{2}-\mathrm{d} \varphi_{3}\right) \\
& \mathrm{dX}=(\sqrt{ } 6 / 4)\left(-\mathrm{d} \varepsilon_{11}+\mathrm{d} \varepsilon_{12}-\mathrm{d} \varepsilon_{13}-2 \mathrm{~d} \varepsilon_{23}-\mathrm{d} \varphi_{2}-\mathrm{d} \varphi_{3}\right) \\
& \mathrm{dX}_{3}=(\sqrt{ } 6 / 4)\left(\mathrm{d} \varepsilon_{11}+\mathrm{d} \varepsilon_{12}-\mathrm{d} \varepsilon_{13}+2 \mathrm{~d} \varepsilon_{23}-\mathrm{d} \varphi_{2}-\mathrm{d} \varphi_{3}\right) \\
& \mathrm{dX} X_{4}=(\sqrt{ } 6 / 4)\left(-\mathrm{d} \varepsilon_{11}+\mathrm{d} \varepsilon_{12}+\mathrm{d} \varepsilon_{13}+2 \mathrm{~d} \varepsilon_{23}+\mathrm{d} \varphi_{2}-\mathrm{d} \varphi_{3}\right) \\
& \mathrm{dY}_{1}=(\sqrt{ } 6 / 4)\left(\mathrm{d} \varepsilon_{22}+\mathrm{d} \varepsilon_{12}-2 \mathrm{~d} \varepsilon_{13}+\mathrm{d} \varepsilon_{23}-\mathrm{d} \varphi_{1}+\mathrm{d} \varphi_{3}\right) \\
& \mathrm{dY}_{2}=(\sqrt{6} 6 / 4)\left(-\mathrm{d} \varepsilon_{22}+\mathrm{d} \varepsilon_{12}+2 \mathrm{~d} \varepsilon_{13}+\mathrm{d} \varepsilon_{23}-\mathrm{d} \varphi_{1}+\mathrm{d} \varphi_{3}\right) \\
& \mathrm{dY}_{3}=(\sqrt{ } 6 / 4)\left(\mathrm{d} \varepsilon_{22}+\mathrm{d} \varepsilon_{12}+2 \mathrm{~d} \varepsilon_{13}-\mathrm{d} \varepsilon_{23}+\mathrm{d} \varphi_{1}+\mathrm{d} \varphi_{3}\right) \\
& \mathrm{dY}_{4}=(\sqrt{ } 6 / 4)\left(-\mathrm{d} \varepsilon_{22}+\mathrm{d} \varepsilon_{12}-2 \mathrm{~d} \varepsilon_{13}-\mathrm{d} \varepsilon_{23}+\mathrm{d} \varphi_{1}+\mathrm{d} \varphi_{3}\right)
\end{aligned}
$$

An actual crystal rotation is carried out as follows.

$$
\begin{gathered}
\mathrm{d} \varphi_{1}=\mathrm{d} \varepsilon_{22}+2 \mathrm{~d} \varepsilon_{12}-2 \mathrm{~d} \varepsilon_{13}-\mathrm{d} \varepsilon_{23} \\
\mathrm{~d} \varphi_{2}=-\mathrm{d} \varepsilon_{11}-\mathrm{d} \varepsilon_{13} \\
\mathrm{~d} \varphi_{3}=\mathrm{d} \varepsilon_{12}-2 \mathrm{~d} \varepsilon_{23}
\end{gathered}
$$

\section{Experimental Results}

\subsection{Fcc Metal}

According to the model in 2.1, Taylor factor $M$ value is calculated as $\Gamma$ itself, i.e. the minimum total slip amount in (2). Figure 4 shows onset (minimum) isosurface of the $M$ value in the ODF of fcc metal under cold rolling. Figure 5(1) is isosurface of $\mathrm{M}=1.81$ in the ODF coordinates and Figure 5(2) shows 2D perspective of it through $\Phi$ axis where band of (001) $[0 \overline{1} 0]$ is apparent, and other spots are all $\{100\}<001>$ cube. Thus in fcc metal onset (minimum) isosurface of Taylor factor $\mathrm{M}$ value under cold rolling solely indicates the cube orientation.

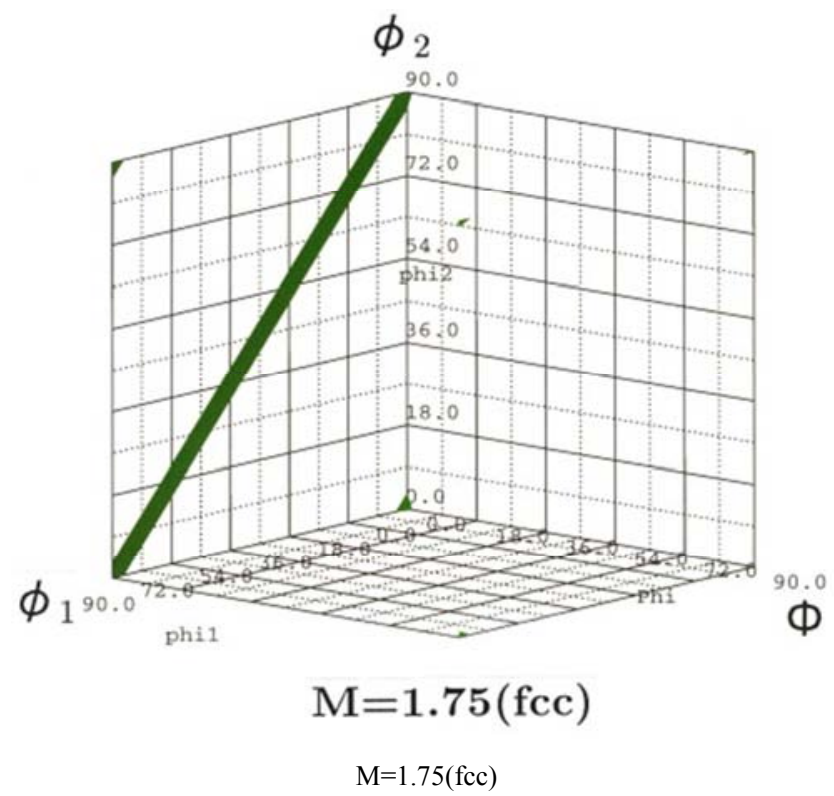

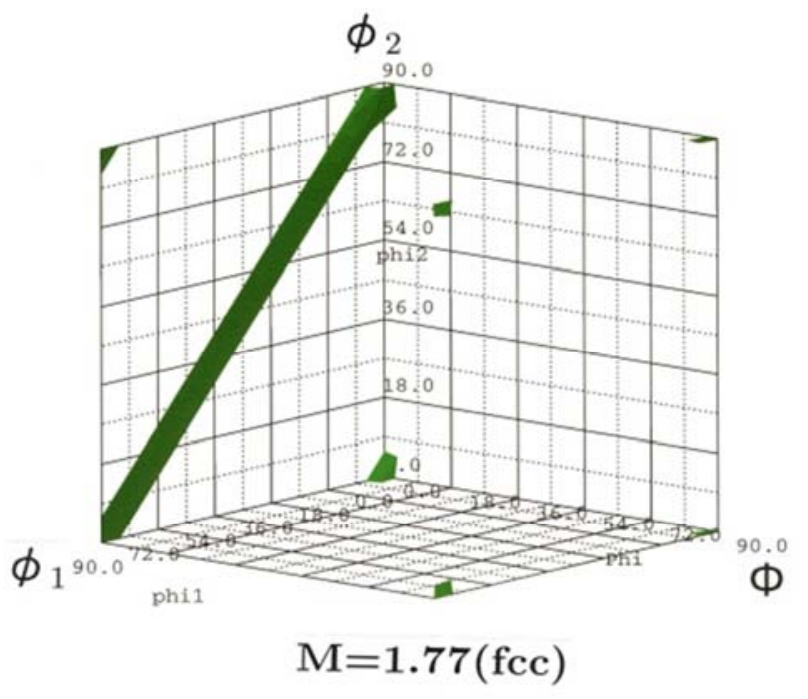

$\mathrm{M}=1.77$

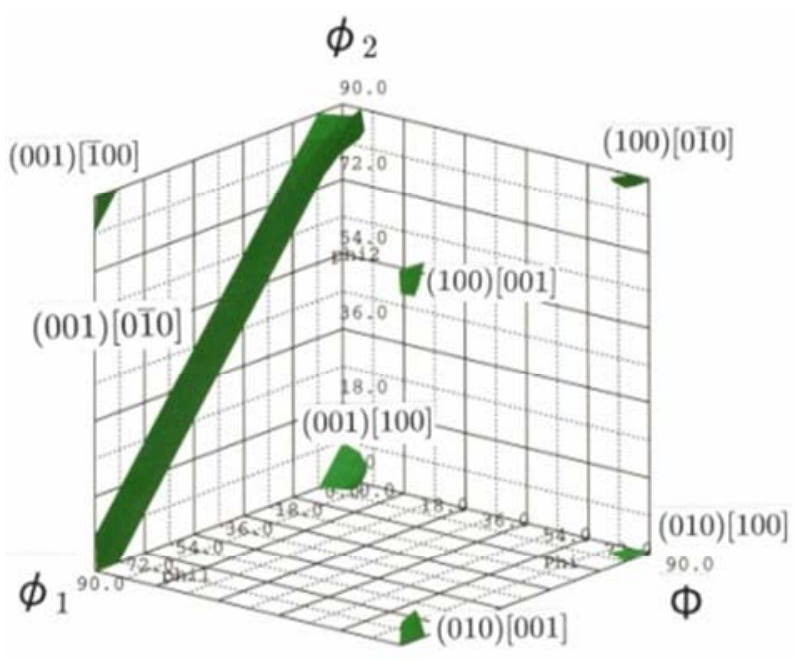

$$
\begin{gathered}
\mathrm{M}=1.81 \text { (fcc) } \\
\mathrm{M}=1.81(\mathrm{fcc})
\end{gathered}
$$

Figure 4. Initial (Minimum) isosurface of Taylor factor $M$ value in the $O D F$ coordinates of fcc metal under cold rolling.

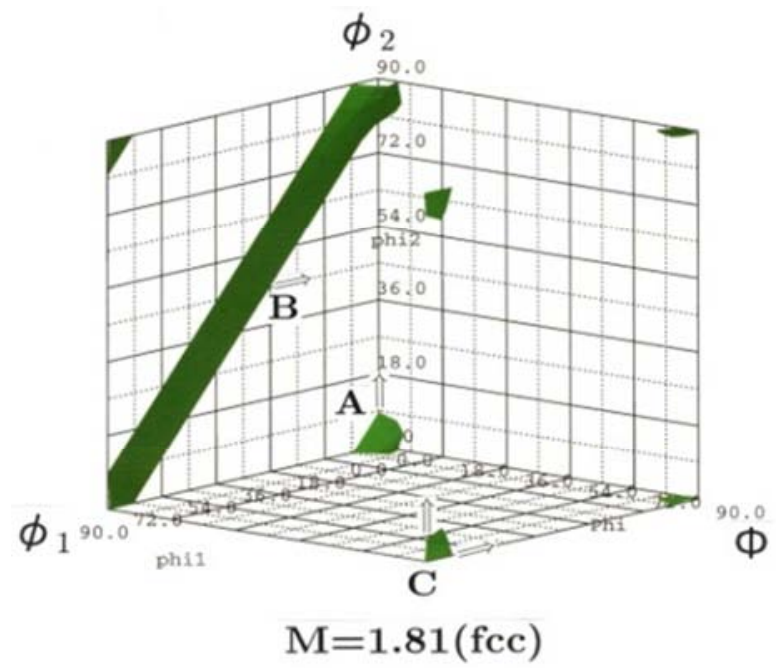

$\mathrm{M}=1.81(\mathrm{fcc})$. 


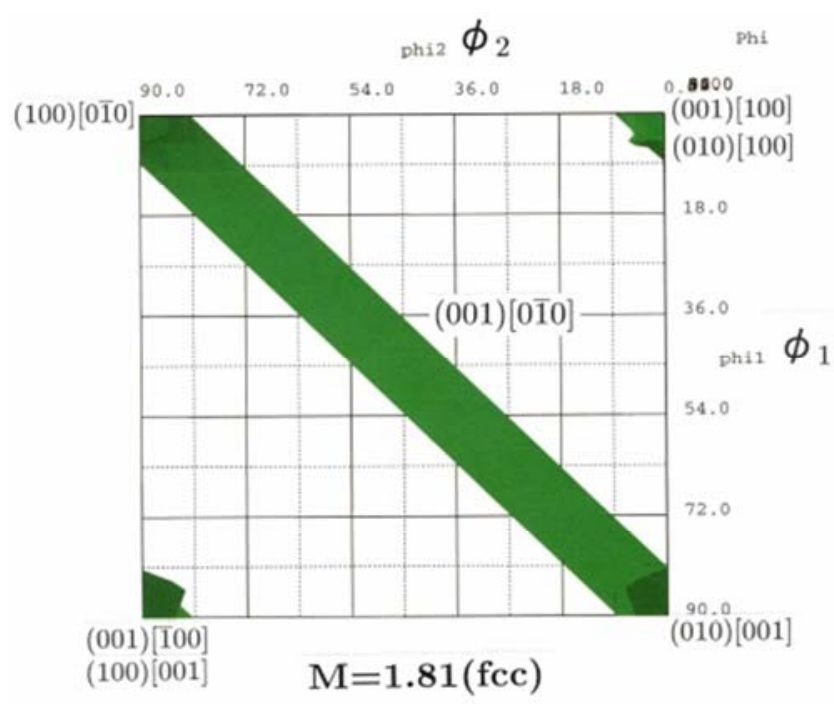

$\mathrm{M}=1.81(\mathrm{fcc})$ (perspective)

\subsection{Bcc Metal}

According to the model, Figure 6 demonstrates how final stable rolling orientation in bcc $(11118)[\overline{4} \overline{4} 11]$ is derived from initial orientation $\left(\begin{array}{lll}1 & 1 & 2\end{array}\right)\left[\begin{array}{lll}\overline{1} & \overline{1} & 1\end{array}\right]$ with increase of strain. At each strain the model selects one solution among the 72 cases composed of 24 ones each belonging to any one of $\mathrm{X}, \mathrm{Y}$ and $\mathrm{Z}$ group. In this Figure, it may be noted i) how the model gains the orientation with strain by way of selecting one of the partitions $\{ \pm X\},\{ \pm Y\}$ and $\{ \pm Z\}$ independently in the rotational symmetrical system and ii) how it holds a continuous value of $\Gamma$ (gamma), the minimum total slip amount by way of (2) through the three $\mathrm{X}, \mathrm{Y}$ and $\mathrm{Z}$ group, so smoothly as not to give such a fatal discontinuity in the value of $\Gamma$ (gamma) at each strain as to lose the symmetry in the system. This is in reasonable accord with an expectation that amount of the external work by applied force to material shall be continuously changed with strain.

Figure 5. Isosurface of Taylor factor $M=1.81$ in the $O D F$ coordinates of fcc metal under cold rolling.

\begin{tabular}{|c|c|c|c|c|c|c|c|c|c|c|c|}
\hline \multirow{2}{*}{\multicolumn{3}{|c|}{ initial }} & phi2 & Phi & phil & \multicolumn{3}{|c|}{ ND } & \multicolumn{3}{|c|}{$\mathrm{RD}$} \\
\hline & & & & 35.26 & 90.00 & 47 & & 0.82 & .5 & -0.58 & 0.58 \\
\hline strain & mode & gamma & phi2 & Phi & phil & \multicolumn{3}{|c|}{ ND } & \multicolumn{3}{|c|}{$\mathrm{RD}$} \\
\hline 0.069 & $\mathrm{Y}$ & 3.77 & 45.00 & 37.13 & 90.00 & 0.43 & 0.43 & 0.80 & -0.56 & -0.56 & 0.60 \\
\hline 0.139 & $\mathrm{Y}$ & 3.74 & 45.00 & 39.43 & 90.00 & 0.45 & 0.45 & 0.77 & -0.55 & -0.55 & 0.64 \\
\hline 0.208 & Y & 3.69 & 45.00 & 42.24 & 90.00 & 0.48 & 0.48 & 0.74 & -0.52 & -0.52 & 0.67 \\
\hline 0.277 & $\mathrm{Y}$ & 3.59 & 45.00 & 45.66 & 90.00 & 0.51 & 0.51 & 0.70 & -0.49 & -0.49 & 0.72 \\
\hline 0.347 & 2 & 3.50 & 45.00 & 49.50 & 90.00 & 0.54 & 0.54 & 0.65 & -0.46 & -0.46 & 0.76 \\
\hline 0.416 & Z & 3.66 & 45.00 & 52.54 & 90.00 & 0.56 & 0.56 & 0.61 & -0.43 & -0.43 & 0.79 \\
\hline 0.485 & Z & 3.74 & 45.00 & 54.91 & 90.00 & 0.58 & 0.58 & 0.57 & -0.41 & -0.41 & 0.82 \\
\hline 0.555 & Z & 3.77 & 45.00 & 56.75 & 90.00 & 0.59 & 0.59 & 0.55 & -0.39 & -0.39 & 0.84 \\
\hline 0.624 & Z & 3.78 & 45.00 & 58.15 & 90.00 & 0.60 & 0.60 & 0.53 & -0.37 & -0.37 & 0.85 \\
\hline 0.693 & Z & 3.78 & 45.00 & 59.22 & 90.00 & 0.61 & 0.61 & 0.51 & -0.36 & -0.36 & 0.86 \\
\hline 0.762 & $\mathrm{X}$ & 3.77 & 45 & 60.04 & 90.00 & 0.61 & 0.61 & 0.50 & -0.35 & -0.35 & 0.87 \\
\hline 0.832 & Z & 3.76 & 45.00 & 60.66 & 90.00 & 0.62 & 0.62 & 0.49 & -0.35 & -0.35 & 0.87 \\
\hline 0.901 & $\mathrm{X}$ & 3.75 & 45.00 & 61.13 & 90.00 & 0.62 & 0.62 & 0.48 & -0.34 & -0.34 & 0.88 \\
\hline 0.970 & Z & 3.74 & 45.00 & 61.49 & 90.00 & 0.62 & 0.62 & 0.48 & -0.34 & -0.34 & 0.88 \\
\hline 1.040 & Z & 3.73 & 45.00 & 61.77 & 90.00 & 0.62 & 0.62 & 0.47 & -0.33 & -0.33 & 0.88 \\
\hline 1.109 & Z & 3.72 & 45.00 & 61.97 & 90.00 & 0.62 & 0.62 & 0.47 & -0.33 & -0.33 & 0.88 \\
\hline 1.178 & $\mathrm{X}$ & 3.72 & 45.00 & 62.13 & 90.00 & 0.63 & 0.63 & 0.47 & -0.33 & -0.33 & 0.88 \\
\hline 1.248 & Z & 3.72 & 45.00 & 62.25 & 90.00 & 0.63 & 0.63 & 0.47 & -0.33 & -0.33 & 0.89 \\
\hline 1.317 & $\mathrm{X}$ & 3.71 & 45.00 & 62.34 & 90.00 & 0.63 & 0.63 & 0.46 & -0.33 & -0.33 & 0.89 \\
\hline 1.386 & Z & 3. & 45.00 & 62. & 0 & 0.63 & 0.63 & 0.46 & -0.33 & -0.33 & 0.89 \\
\hline 1.456 & Z & 3.71 & 45.00 & 62.47 & 90.00 & 0.63 & 0.63 & 0.46 & -0.33 & -0.33 & 0.89 \\
\hline 1.525 & Z & 3.71 & 45.00 & 62.51 & 90.00 & 0.63 & 0.63 & 0.46 & -0.33 & -0.33 & 0.89 \\
\hline 1.594 & Z & 3.71 & 45.00 & 62.54 & 90.00 & 0.63 & 0.63 & 0.46 & -0.33 & -0.33 & 0.89 \\
\hline 1.664 & Z & 3.71 & 45.00 & 62.56 & 90.00 & 0.63 & 0.63 & 0.46 & -0.33 & -0.33 & 0.89 \\
\hline 1.733 & Z & 3.70 & 45.00 & 62.58 & 90.00 & 0.63 & 0.63 & 0.46 & -0.33 & -0.33 & 0.89 \\
\hline 1.802 & Z & 3.70 & 45.00 & 62.59 & 90.00 & 0.63 & 0.63 & 0.46 & -0.33 & -0.33 & 0.89 \\
\hline 1.872 & Z & 3.70 & 45.00 & 62.60 & 90.00 & 0.63 & 0.63 & 0.46 & -0.33 & -0.33 & 0.89 \\
\hline 1.941 & $\mathrm{Y}$ & 3.70 & 45.00 & 62.61 & 90.00 & 0.63 & 0.63 & 0.46 & -0.33 & -0.33 & 0.89 \\
\hline 2.010 & Z & 3.70 & 45.00 & 62.61 & 90.00 & 0.63 & 0.63 & 0.46 & -0.33 & -0.33 & 0.89 \\
\hline
\end{tabular}

Figure 6. Simulation results by the model how a final stable rolling orientation (11 118$)[\overline{4} \overline{4} 11]$ of bcc metal is derived from initial orientation (1 112$)\left[\begin{array}{lll}\overline{1} & \overline{1} & 1\end{array}\right]$ with increase of strain. 
As shown in Figure 6, due to symmetry [1], crystal selects one of X [100], Y [010] and Z [001] groups (mode) in each strain, holding a continuous value of $\Gamma$ (gamma), the minimum total slip amount by way of (2) which is a conservation quantity [2] in the three $\mathrm{X}, \mathrm{Y}$ and $\mathrm{Z}$ symmetry group and even Taylor factor $M$ value itself of the material. In the column of "mode" in Figure 6, it is cleared how the model selects one solution among the 72 cases composed of 24 ones each belonging to any one of $\mathrm{X}, \mathrm{Y}$ and $\mathrm{Z}$ group at each strain.

Figure 7 illustrates by the model dynamically how orientations in ODFs map at rolling ratio of (a) $6 \%$, (b) $38 \%$, (c) $62 \%$ and (d) $95 \%$ are rigorously accumulated from initial random ones with strain in rolling by the present model and consequently the three stable orientations of bcc metal in rolling [4]- [9] such as $\{112\}\langle 110\rangle,\{11118\}\langle 4411\rangle$ and $\{100\}\langle 011\rangle$ are attained as drawn on Figure $7(d)$.
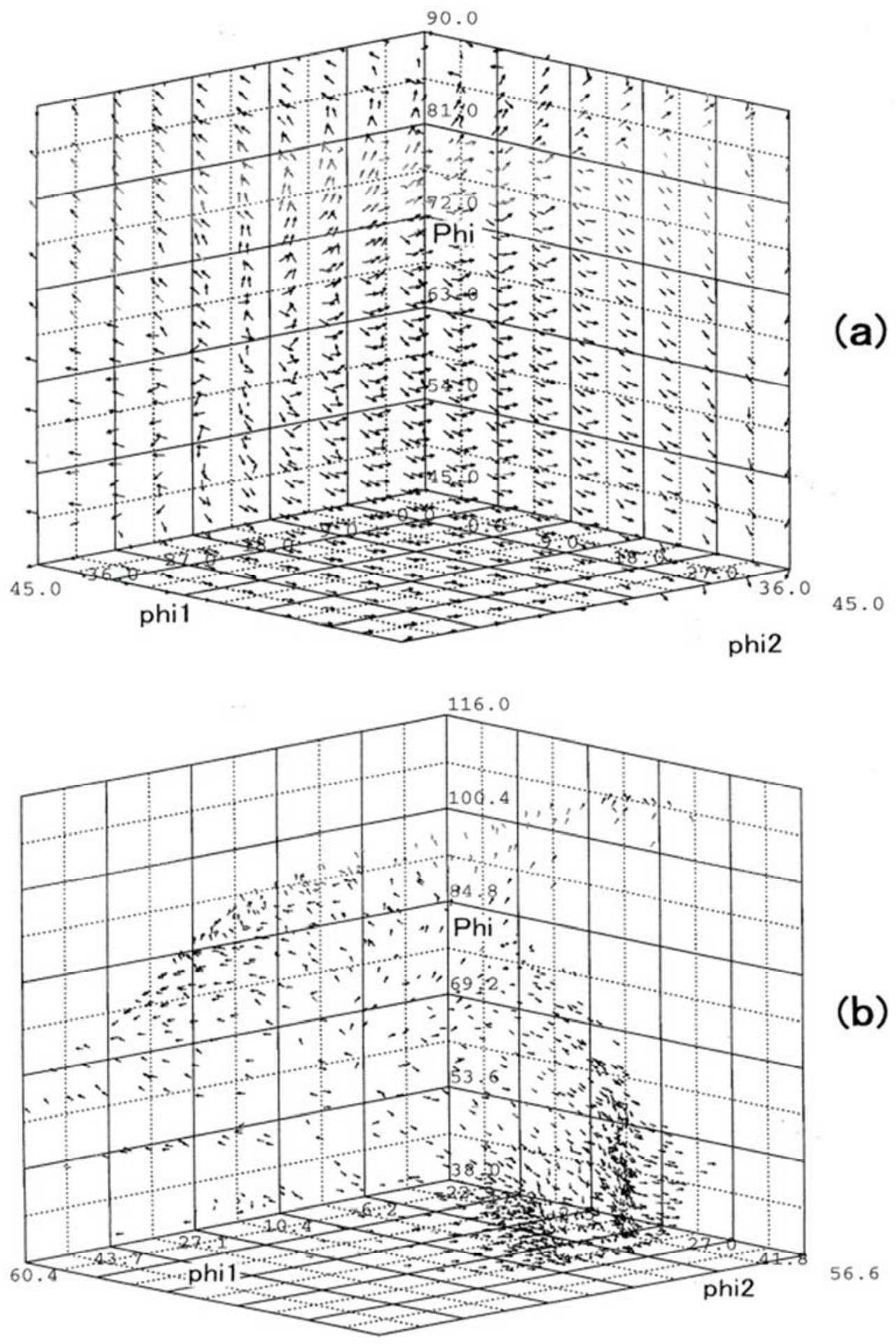

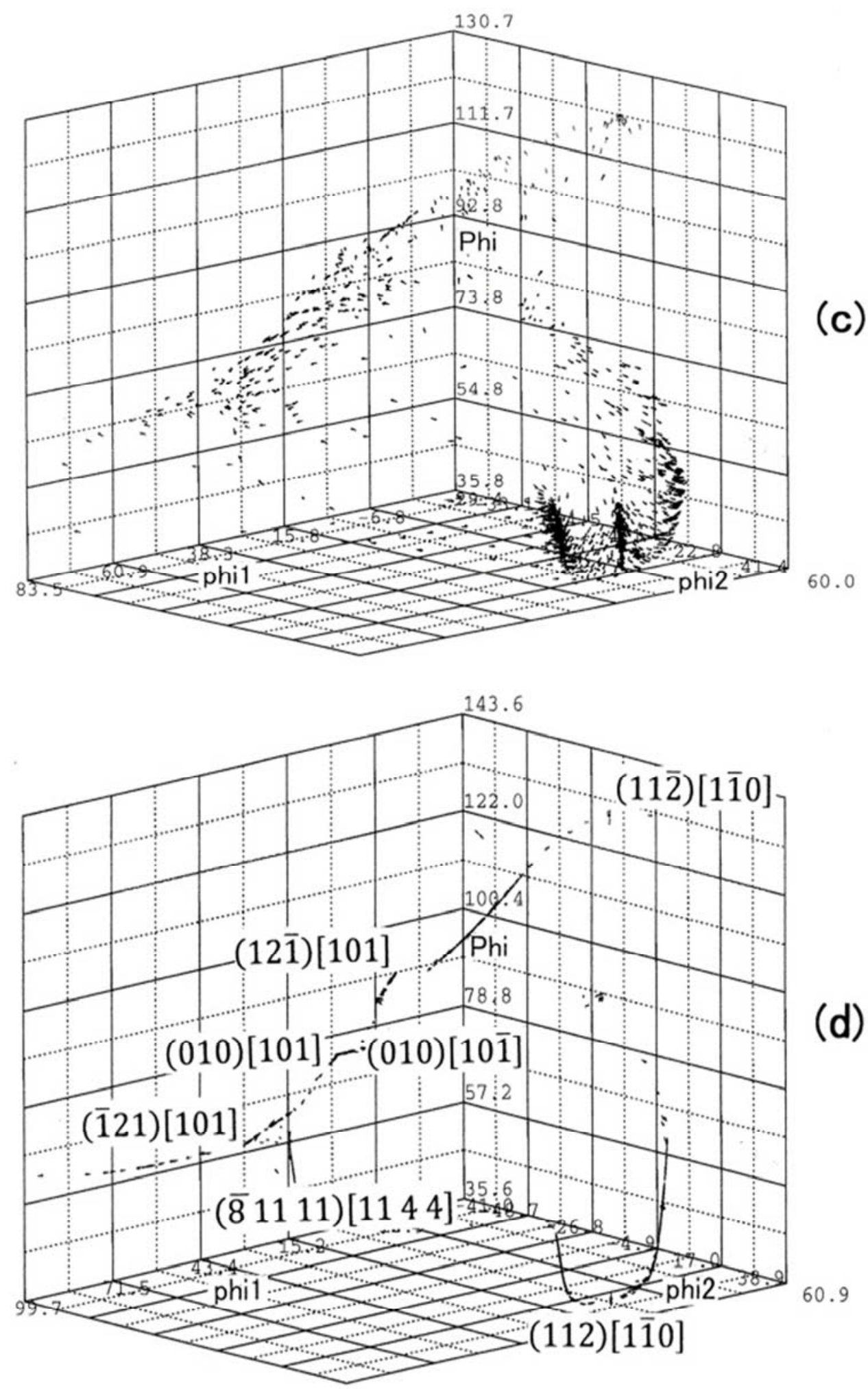

Figure 7. A visible demonstration by the model how and when the rolling orientation in bcc metal changes and appears in the ODFs map which shows the orientations at rolling ratio of (a) $6 \%,(b) 38 \%,(c) 62 \%$ and $(d) 95 \%$.

\section{Discussion}

\subsection{Fec Metal}

As shown in Figure 4 and Figure 5 the isosurface of distribution of Taylor factor $M$ value in the ODF coordinates of fcc metal under cold rolling is characterized by existence of the cube structure at onset (minimum) of the isosurface. The cube structure gradually develops and forms a skeleton of distribution of the $\mathrm{M}$ value in cold rolling of fcc metal. This 
cube $M$ value may be plausibly related with the cube structure in recrystallized fcc metal. Figure 5(1) shows notation A, B and $\mathrm{C}$ of representative $\{100\}<001>$ spots at the onset of Taylor factor $\mathrm{M}=1.81 \mathrm{in}$ fcc metal and Figure 5(2) shows details of the $\mathrm{M}$ value by $2 \mathrm{D}$ perspective of it through $\Phi$ axis. From those figures, Figure 8 is plotted for orientation change from the cube $\{100\}<001>$ where orientation changes gradually $\{100\}<001>\rightarrow\{100\}<016>\rightarrow\{100\}<013>\rightarrow\{100\}<012>\rightarrow\{$ $100\}<023>\rightarrow\{100\}<0,9,11>$ with decrease of $\varphi_{1}$ at spot "B" in Figure 5(1).

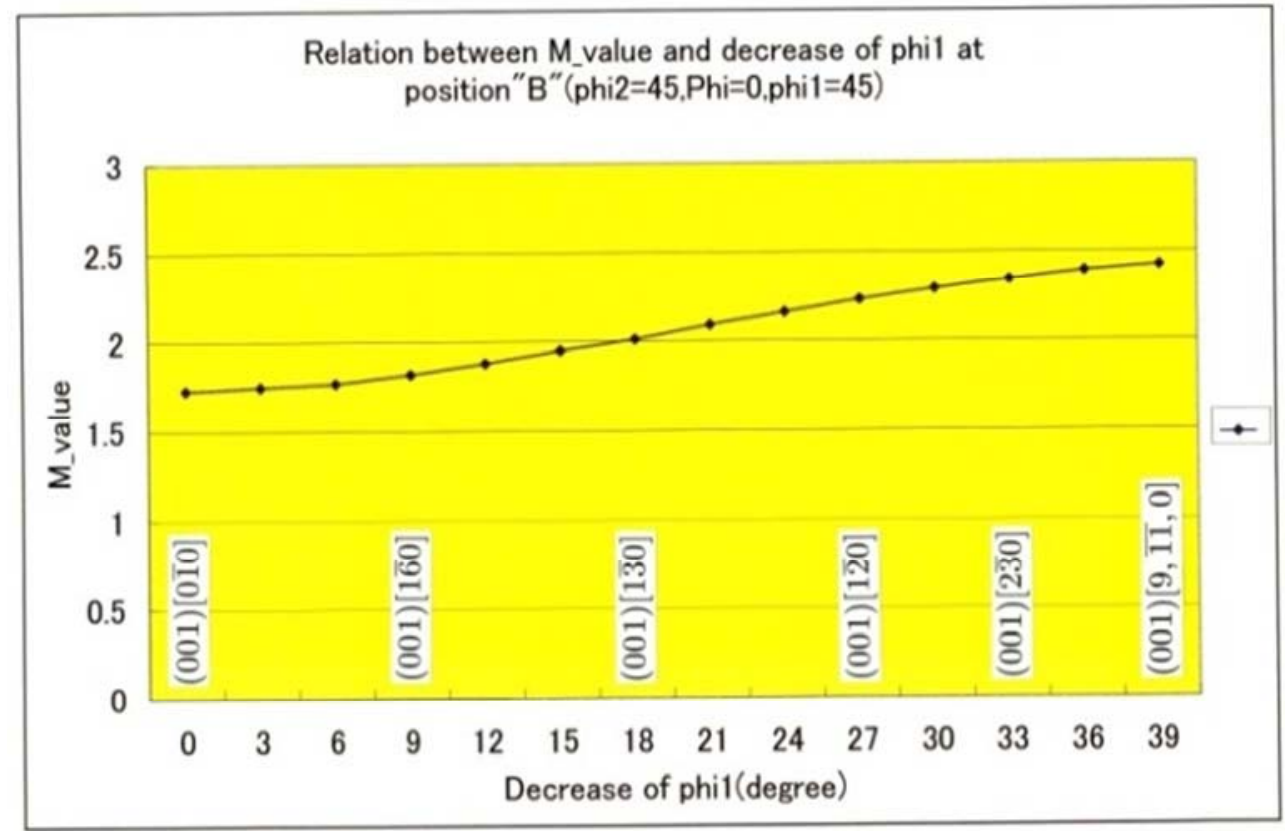

Figure 8. Change of Taylor factor M value with decrease of $\varphi_{1}$ from cube structure $\{100\}<001>$ at point B in the ODF coordinates of Figure $5(1)$ of fcc metal under cold rolling.

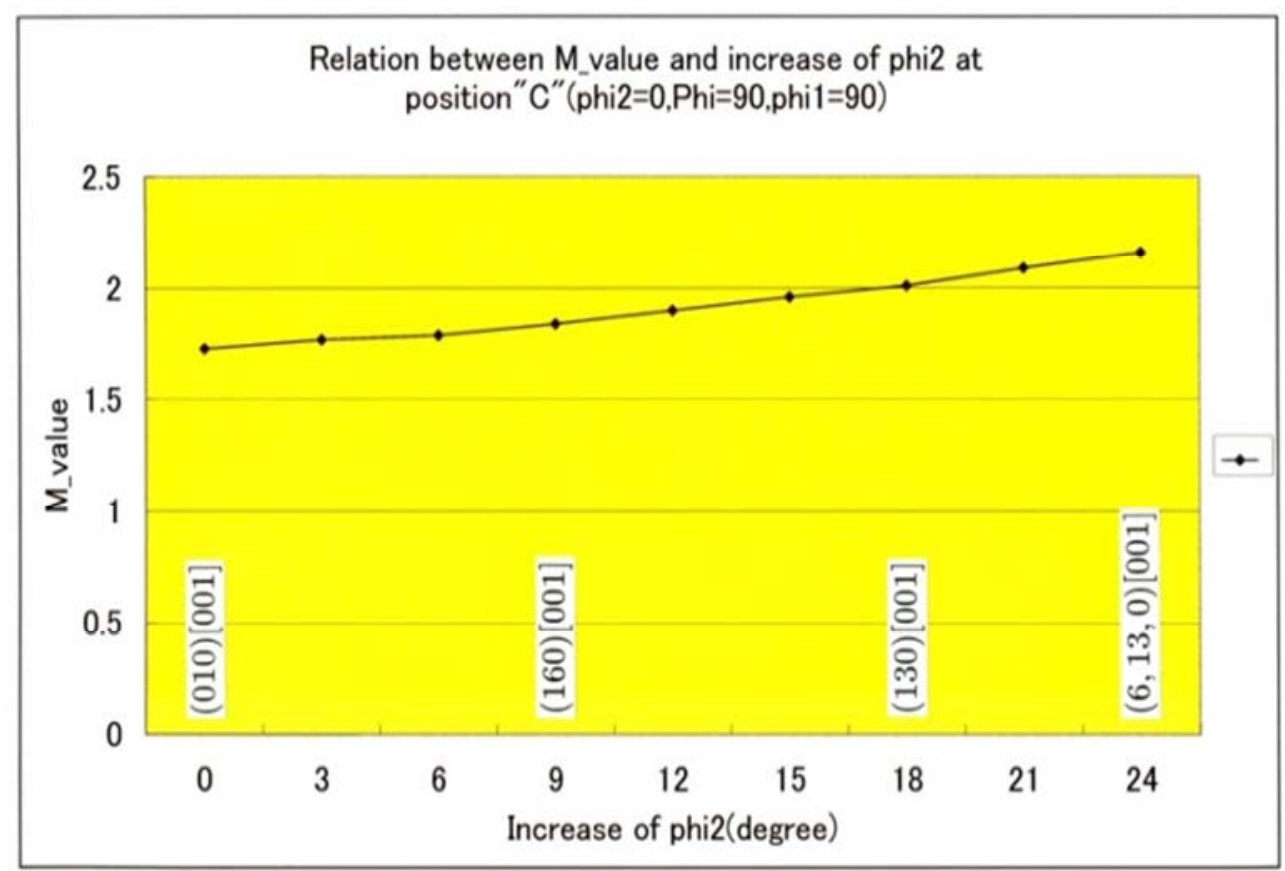

Figure 9. Change of Taylor factor $M$ value with increase of $\varphi_{2}$ from cube structure $\{100\}<001>$ at point $C$ in the ODF coordinates of Figure $5(1)$ of fcc metal under cold rolling.

Figure 9 is plotted for orientation change from the cube $\{100\}<001>$ where orientation changes gradually $\{100\}<001>\rightarrow\{016\}<100>\rightarrow\{013\}<100>\rightarrow\{0,6,13\}<100>$ with increase of $\varphi_{2}$ at spot " $C$ " in Figure 5(1).
Interesting is the reports [10]- [20] that there were many observed recrystallized grains of indiscrete orientations in fcc metal after cold rolling such as $\{100\}<013>,\{100\}<012>$ and $\{015\}<100>,\{013\}<100>,\{012\}<100>,\{034\}<100>$, etc. 
other than the cube $\{100\}<001>$ orientation. These coincide with orientations in range of Figure 8 and Figure 9. Recently with development of observation with EBSD, the cube-family orientation grains are known to grow in clusters around the cube grain [12-14].

On the other hand, similar hypothesis [19] that "the minimum possible" Taylor factor $M$ value of fcc metal in cold rolling at cube orientation with lowest dislocation density, shall encourage recovering from cold state more easily, could be linked with the present study. The present study may plausibly explain why the cube and the cube-family coexist in fcc recrystallized metal by means of low Taylor factor in addition to a fact that the $\{100\}<0 \mathrm{kl}>$ grains in hot band inevitably rotate toward the cube by cold rolling [12]- [15].

\subsection{Bcc Metal}

According to the model, Figure 6 illustrates how final stable rolling orientation in bcc (11 11 8) [ $\overline{4} \overline{4} 11]$ is derived from initial orientation (1 112$)\left[\begin{array}{lll}\overline{1} & \overline{1} & 1\end{array}\right]$ with increase of strain sequentially selecting one among the 72 cases composed of 24 ones each belonging to any one of $\mathrm{X}, \mathrm{Y}$ and $\mathrm{Z}$ group. By the model, it has been already shown that stable (112)[1]10] of bcc metal is derived from initial orientation (111) [110] in similar way [21], but at that time other stable orientation had not been ascertained yet.

As in Figure 6, $\Gamma$ (gamma), i.e. the minimum total slip amount, by way of (2) in the three $\mathrm{X}, \mathrm{Y}$ and $\mathrm{Z}$ group, is not constant and changes but so gradually as not to give such a fatal discontinuity in the value of $\Gamma$ (gamma) as to lose the symmetry in the system. As generally known, even in the stable system composed of extreme symmetry, it can lose the symmetry immediately and transiently when exposed to external forces or other physical energy. Even in this case, however, as it is still the orthodoxy accepted by the majority, if a break of symmetry is so small where the symmetry recovers immediately and sustain still continuously the original symmetric state that the symmetry may still give birth to forceful analytical means to the phenomenon [22]-[24]. Besides example of Figure 6, it is supposed that such phenomena by the model may happen through the whole orientation as shown in Figure 7.

Similar model on fcc metal as described in model 2.1 requires 24 cases in total for one solution where two combinations of five slips are accepted by the Taylor's formidable restriction rule of the five slips similarly as in Figure 3 on $\{111\}$ planes on every four directions of $\langle 110\rangle$ for each group of X, Y and Z principal axes [25]- [27]. A ratio of the $24(=2 \times 4 \times 3)$ of fcc metal to the 72 of bcc metal which has three intersections of $\{110\}$ planes, implies a ratio of one $\{111\}$ slip plane on direction $\langle 110\rangle$ of fcc metal to at least three slip planes $\{110\},\{112\}$ and $\{123\}$ on direction of $\langle 111\rangle$ in the pencil glide theory of bcc metal [4] [28] [29].

\section{Conclusion}

The principal axes of $X$ [100], Y [010] and Z [001] are perpendicular to each other as the three orbits of
$\{ \pm X\},\{ \pm Y\}$ and $\{ \pm Z\}$ by a rotational symmetry of mathematical group theory in such way that component $X$ is not related to $\mathrm{Y}$ or $\mathrm{Z}$ one another whichever. There is a conservation quantity in the symmetry. As Taylor proved, crystal rotates so that slips occur associating themselves with the minimum total slip amount. The minimum total slip amount in crystal by Taylor corresponds to both the conservation quantity in the rotational symmetry of cubic crystal and even Taylor factor $M$ value itself of the material. It will be demonstrated in this study that these approaches are useful for both face centered cubic (fcc) metal and body centered cubic (bcc) metal.

1. In fcc metal, distributions in 3D ODF coordinates for Taylor factor $\mathrm{M}$ value, i.e. the minimum total slip amount under cold rolling was calculated based on Taylor's formidable restriction rule of the five slips. Main results are as follows. In fcc metal, orientation at onset (minimum) of the $M$ value shows the cube $\{100\}<001>$ and the $M$ value increases gradually $\{100\}<001>\rightarrow\{100\}<016>\rightarrow\{100\}<013>\rightarrow\{100\}<012>\rightarrow$ $\{100\}<023>\rightarrow\{100\}<0,9$, 11> with decrease of $\varphi_{1}$ or $\{100\}<001>\rightarrow\{016\}<100>\rightarrow\{013\}<100>\rightarrow\{0,6,13\}$ $<100>$ with increase of $\varphi_{2}$, most of which were experimentally reported as indiscrete recrystallized orientations with lowest dislocation density named the cluster composed of cube and cube-family in fcc metal.

2. In bcc metal, an intersection of two kinds of $\{110\}$ planes from the three ones composed of $\{110\},\{101\}$ and $\{011\}$ is chosen. Based on the rotational symmetry of the principal axes of X [100], Y [010] and Z [001], 72 possible combinations of the five slips on $\{110\}$ planes based on Taylor's formidable restriction rule of the five slips are calculated among three kinds of intersections of two $\{110\}$ planes on $\langle 111\rangle$ direction in bcc metal. Crystal rotation is carried out by only one solution among the 72 by the minimum total slip amount at every strain and simulates properly lengthy of accumulated researcher's experimental results such as the three stable orientations of bcc metal in rolling $\{112\}\langle 110\rangle,\{11118\}\langle 4411\rangle$ and $\{100\}\langle 011\rangle$.

\section{References}

[1] M. A. Armstrong, Groups and Symmetry (Springer), 2010, pp. 104-165.

[2] E. Noether, Nachr. Gesellsch. Wiss. Goettingen, vol. 2, 1918, pp. 235.

[3] G. I. Taylor, "Plastic Strain in Metals", J. Inst. Metals, vol. 62, 1938, pp. 307-324.

[4] Q. Xie, A. Van Bael, J. Sidor, J. Moerman and P. Van Houtte, Acta Mater., vol. 69, 2014, pp. 175.

[5] M. Takenaka, Y. Shingaki, T. Imamura and Y. Hayakawa,"Influence of carbon content on cold rolling and recrystallization texture in polycrystal $3 \% \mathrm{Si}-\mathrm{Fe}$ ", Proc. ICOTOM 17, 2016, Ser. No. 12042.

[6] M. Gensamer and R. F. Mehl, Trans. AIME, vol. 120, 1936, pp. 277.

[7] I. L. Dillamore and H. Katoh, Metal Science, vol. 8, 1974, pp. 73. 
[8] D. Rollet and S. I. Wright, Texture and Anistropy (Cambridge University Press), 1998, pp. 179-201.

[9] X. R. Sun, H. Z. Wang, P. Yang and W. M. Mao, Acta Metall. Sin., vol. 50, 2014, pp. 387-394.

[10] Y. Zhang, D. Juul Jensen and A. Godfrey Mat. Sci. Forum, vol. 715-716, 2012, pp. 329-332.

[11] L. Lapeire, J. Sidor, E. Martinez Lombardia, K. Verberken, I De Graeve, H. terryn and L Al Kestens,"Texture comparison between cold rolled and cryogenically rolled pure copper", Proc. ICOTOM 17, 2016, Ser. No. 12016.

[12] O. Daaland and E. Nes, "Origin of Cube Texture during Hot Rolling of Commercial Al-Mn-Mg Alloys", Acta mater., vol. 44, No. 4, 1996, pp. 1389-1411.

[13] J. Hjelen J, R. Orsund and E. Nes, "On the Origin of recrystallization Textures in Aluminum", Acta metal. mater., vol. 39, No. 7, 1991, pp. 1377-1404.

[14] H. Inagaki and A. Umezawa, "Origin of Cube Recrystallization Textures in Heavily Rolled High Purity Al", Materials Science Forum, vol. 495-497, 2005, pp. 1273 -1278.

[15] M. Koizumi, H. Okudaira and H. Inagaki, "Influenceof Fe Content on Recrystallization, Grain Growth and Recrystallization Textures inAl-Mg Alloys", Proc. ICOTOM12, 1999, pp. 860- 865 .

[16] D. M. Liu, W, P, Liu, X. Liu and M. L. Zhou," Texture Control in Silver", Materials Science Forum, vol. 495-497, 2005, pp. 725-730.

[17] N. Rajmohan and J. A. Szpunar, "Stored Energy in Can Body Aluminum Alloy after Cold Rolling and Stress Relieving",
Materials Science and Technology, vol. 15, 1999, pp. 1259-1265.

[18] O. Engler, "Recrystallization Textures in Copper-Manganese Alloys", Acta mater, vol. 49, 2001, pp. 1237-1247.

[19] A. A. Ridha and W. B. Hutchinson,"Recrystall- ization Mechanisms and the Origin of Cube Texture in Copper", Acta Metal, vol. 30, 1982, pp. 1929-1939.

[20] D. Juul Jensen, F. Lin and Y. Zhang, Mat. Sci. Forum, vol. 753, 2013, pp. 37-41.

[21] H. Masui,"Simulations of texture formation of body-centered-cubic metals by three kinds of intersections of two $\{110\}$ slip planes”, Proc. ICOTOM17, 2016, Ser. No. 12010.

[22] R. Arita, K. Kusakabe, K. Kuroki and H. Aoki, J. Phys. Soc. Jpn, vol. 66, 1997, pp. 2086.

[23] X. G. Wen, Quantum Field Theory of Many Body System (Oxford University Press), 2004.

[24] Y. Kazama, Symmetry and conservation quantity (Science Ltd, Japan), 2008, pp. 26-32.

[25] H. Masui, "Simulation for F. c. c. Deformation Texture by Modified Pencil Glide Theory", Acta mater, vol.47, No. 17, 1999, pp. 4283-4298.

[26] H. Masui, Materials Science Forum, vol. 495- 497, 2005, pp. 971.

[27] H. Masui, Ceramics Trans. vol. 201, 2008, pp. 489.

[28] E. A. Calnan and C. J. B. Clews, Phil. Mag., vol. 42, 1951, pp. 616.

[29] A. D. Rollet and U. F. Kocks, Proc. ICOTOM 8, 1988, pp. 375. 